Mathematical Modelling and Analysis

Volume 16 Number 3, September 2011, 390-400

Doi:10.3846/13926292.2011.601770

(C) Vilnius Gediminas Technical University, 2011
Publisher: Taylor\&Francis and VGTU

www.tandfonline.com/loi/tmma20

Online ISSN: $1648-3510$

Print ISSN: 1392-6292

\title{
Some Multiplicity Results to the Existence of Three Solutions for a Dirichlet Boundary Value Problem Involving the $p$-Laplacian*
}

\author{
Shapour Heidarkhani ${ }^{a, b}$ and Ghasem Alizadeh Afrouzi ${ }^{c}$ \\ ${ }^{a}$ Razi University \\ 67149 Kermanshah, Iran \\ ${ }^{b}$ Institute for Research in Fundamental Sciences \\ P.O. Box: 19395-5746, Tehran, Iran \\ ${ }^{c}$ University of Mazandaran \\ 47416-1467 Babolsar, Iran \\ E-mail(corresp.): s.heidarkhani@razi.ac.ir \\ E-mail: afrouzi@umz.ac.ir
}

Received July 27, 2010; revised June 17, 2011; published online August 1, 2011

\begin{abstract}
In this paper we prove the existence of two intervals of positive real parameters $\lambda$ for a Dirichlet boundary value problem involving the $p$-Laplacian which admit three weak solutions, whose norms are uniformly bounded with respect to $\lambda$ belonging to one of the two intervals. Our main tool is a three critical points theorem due to G. Bonanno [A critical points theorem and nonlinear differential problems, J. Global Optim., 28:249-258, 2004].
\end{abstract}

Keywords: three solutions, critical point, variational methods, multiplicity results, Dirichlet problem.

AMS Subject Classification: 35J20; 34A15.

\section{Introduction}

The purpose of this paper is to establish the existence of two intervals of positive real parameters $\lambda$ for which the problem

$$
\begin{cases}\Delta_{p} u+\lambda f(x, u)=0 & \text { in } \Omega \\ u=0 & \text { on } \partial \Omega\end{cases}
$$

where $\Delta_{p} u=\operatorname{div}\left(|\nabla u|^{p-2} \nabla u\right)$ is the $p$-Laplacian operator, $\Omega \subset R^{N}(N \geqslant 1)$ is a non-empty bounded open set with smooth boundary $\partial \Omega, p>N, \lambda$ is a positive parameter and $f: \Omega \times R \rightarrow R$ is an $L^{1}$ - Carathéodory function,

\footnotetext{
* This research was in part supported by grant from IPM (No. 89350020).
} 
admits three weak solutions, whose norms are uniformly bounded in respect to $\lambda$ belonging to one of the two intervals.

We recall that a function $f: \Omega \times R \rightarrow R$ is said to be $L^{1}$-Carathéodory if

$\left(\delta_{1}\right) x \rightarrow f(x, t)$ is measurable for every $t \in R$;

$\left(\delta_{2}\right) t \rightarrow f(x, t)$ is continuous for almost every $x \in \Omega$;

$\left(\delta_{3}\right)$ for every $\varrho>0$ there exists a function $l_{\varrho} \in L^{1}(\Omega)$ such that

$$
\sup _{|t| \leqslant \varrho}|f(x, t)| \leqslant l_{\varrho}(x)
$$

for almost every $x \in \Omega$.

We say that $u$ is a weak solution to the problem (1.1) if $u \in W_{0}^{1, p}(\Omega)$ and

$$
\int_{\Omega}|\nabla u(x)|^{p-2} \nabla u(x) \nabla v(x) d x-\lambda \int_{\Omega} f(x, u(x)) v(x) d x=0
$$

for every $v \in W_{0}^{1, p}(\Omega)$.

In recent years, many publications $[1,7,8,9,10,11,12,14]$ have appeared about elliptic problems with Dirichlet boundary conditions which have been used in a great variety of application. For example, Ramaswamy and Shivaji in [14] established the existence of three positive solutions for classes of nondecreasing, $p$-sublinear functions $f$ belonging to $C^{1}([0, \infty))$ for a $p$-Laplacian version of [3], i.e., the problem

$$
\begin{cases}-\Delta_{p} u=\lambda f(u) & \text { in } \Omega \\ u=0 & \text { on } \partial \Omega\end{cases}
$$

where $p>1, \lambda>0$ is a parameter and $\Omega$ is a bounded domain in $R^{N} ; N \geq 2$ with $\partial \Omega$ of class $C^{2}$ and connected. Uniqueness of positive solutions to the problem (1.2) when $p>1$ and $f(u) / u^{p-1}$ is decreasing on $(0,+\infty)$ was obtained in Guo and Webb [11] and Drabek and Hernandez [9]. A natural question is that, whether uniqueness holds under the weaker condition than $f(u) / u^{p-1}$ is decreasing for large $\mathrm{u}$. When $\Omega$ is a ball, Hai and Shivaji [12] showed that the answer is affirmative. However, the approach used in [12] depends on ordinary differential equations techniques and cannot be applied to the case of a general domain. In [7], Ricceri's three critical points theorem [15] has been successfully used to obtain existence of at least three weak solutions to the problem (1.1) in $W_{0}^{1, p}(\Omega)$. In [1], based on Ricceri's three critical points theorem [15] we obtained the existence of an interval $\Lambda \subseteq[0,+\infty[$ and a positive real number $q$ such that for each $\lambda \in \Lambda$ problem

$$
\begin{cases}\Delta_{p} u+\lambda f(x, u)=a(x)|u|^{p-2} u & \text { in } \Omega, \\ u=0 & \text { on } \partial \Omega,\end{cases}
$$

where $\Omega \subset R^{N}(N \geqslant 2)$ is non-empty bounded open set with smooth boundary $\partial \Omega, p>N, \lambda>0, f: \Omega \times R \rightarrow R$ is a continuous function and positive weight function $a(x) \in C(\bar{\Omega})$, admits at least three weak solutions whose norms in 
$W_{0}^{1, p}(\Omega)$ are less than $q$ that we extended the main result of [4] by using of the results of [7] to the general case. In [8], the authors employing Ricceri's three critical points theorem [16] obtained multiple weak solutions for the following BVP

$$
\begin{cases}-\Delta_{p} u=\lambda f(x, u)+\mu g(x, u) & \text { in } \Omega, \\ u=0 & \text { on } \partial \Omega,\end{cases}
$$

where $\Omega \subset R^{N}$ is a non-empty bounded open set with smooth boundary $\partial \Omega$, $p>N, f, g: \Omega \times R \rightarrow R$ are two Carathéodory functions and $\lambda, \mu$ are two positive parameters.

Bonanno in [6] established the existence of two intervals of positive real parameters $\lambda$ for which the functional $\Phi+\lambda \Psi$ has three critical points, whose norms are uniformly bounded with respect to $\lambda$ belonging to one of the two intervals. He illustrated the result for a two point boundary value problem, and here we are interested to illustrate this result to the problem (1.1). Our main result is Theorem 1 that ensures the existence of two intervals $\Lambda_{1}^{\prime}$ and $\Lambda_{2}^{\prime}$ such that, for each $\lambda \in \Lambda_{1}^{\prime} \cup \Lambda_{2}^{\prime}$, the problem (1.1) admits at least three weak solutions whose norms are uniformly bounded with respect to $\lambda \in \Lambda_{2}^{\prime}$. The technique used in our proof has been introduced in [7].

As an immediate consequences of Theorem 1, we obtain Corollary 1, in which the function $f$ has separated variables. The applicability of the result is illustrated by Example 1. Finally, we present the application of Theorem 1 in the ordinary case with $p=2$, that Example 2 illustrates the result.

\section{Main Results}

First we recall for the reader's convenience Theorem 3.1 of $[6]$ (see also $[2,5$, $13,15,16]$ for related results) to transfer the existence of three solutions of the problem (1.1) into the existence of critical points of the Euler functional:

Theorem A ([6, Theorem 3.1]) Let $X$ be a separable and reflexive real Banach space; $\Phi: X \longrightarrow R$ a nonnegative continuously Gâteaux differentiable and sequentially weakly lower semicontinuous functional whose Gâteaux derivative admits a continuous inverse on $X^{*} ; J: X \longrightarrow R$ a continuously Gâteaux differentiable functional whose Gateaux derivative is compact. Assume that there exists $x_{0} \in X$ such that $\Phi\left(x_{0}\right)=J\left(x_{0}\right)=0$ and that

(i) $\lim _{\|x\| \rightarrow+\infty}(\Phi(x)-\lambda J(x))=+\infty$ for all $\lambda \in[0,+\infty[$.

Further, assume that there are $r>0, x_{1} \in X$ such that:

(ii) $r<\Phi\left(x_{1}\right)$,

(iii) $\sup _{x \in \bar{\Phi}^{-1}(]-\infty, r[)} w(x)<\frac{r}{r+\Phi\left(x_{1}\right)} J\left(x_{1}\right)$.

Then, for each

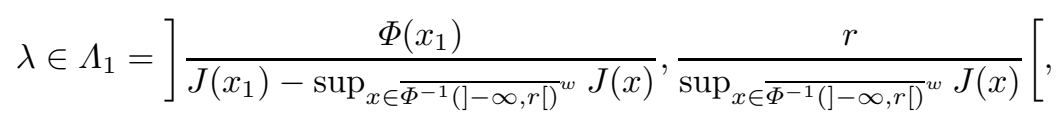


the equation $\Phi^{\prime}(u)-\lambda J^{\prime}(u)=0$ has at least three solutions in $X$ and, moreover, for each $h>1$, there exist an open interval

$$
\Lambda_{2} \subseteq\left[0, h r /\left(r J\left(x_{1}\right) / \Phi\left(x_{1}\right)-\sup _{x \in \Phi^{-1}(-\infty, r[)} w(x)\right)\right]
$$

and a positive real number $\sigma$ such that, for each $\lambda \in \Lambda_{2}$, the equation given above has at least three solutions in $X$ whose norms are less than $\sigma$.

Here and in the sequel, $\mathrm{X}$ will denote the Sobolev space $W_{0}^{1, p}(\Omega)$ with the norm

$$
\|u\|=\left(\int_{\Omega}|\nabla u(x)|^{p} d x\right)^{1 / p} .
$$

Put $F(x, t)=\int_{0}^{t} f(x, \xi) d \xi$ for each $(x, t) \in \Omega \times R$, and

$$
c=\sup _{u \in X \backslash\{0\}} \frac{\max _{x \in \bar{\Omega}}|u(x)|}{\|u\|} .
$$

Since $p>N, X$ is compactly embedded in $C^{0}(\bar{\Omega})$, one has $c<+\infty$. In addition, it is known [18, formula $(6 \mathrm{~b})]$ that

$$
c \leqslant \frac{N^{-1 / p}}{\sqrt{\pi}}\left[\Gamma\left(1+\frac{N}{2}\right)\right]^{1 / N}\left(\frac{p-1}{p-N}\right)^{1-1 / p}[m(\Omega)]^{1 / N-1 / p},
$$

where $\Gamma$ denotes the Gamma function and $m(\Omega)$ is the Lebesgue measure of the set $\Omega$, and equality occurs when $\Omega$ is a ball.

Now, fix $x^{0} \in \Omega$ and pick $r_{1}, r_{2}$ with $0<r_{1}<r_{2}$ such that

$$
S\left(x^{0}, r_{1}\right) \subset S\left(x^{0}, r_{2}\right) \subseteq \Omega
$$

where $S\left(x^{0}, r_{i}\right)$ denotes the ball with center at $x^{0}$ and radius of $r_{i}$ for $i=1,2$. Put

$$
k_{1}=k_{1}\left(N, p, r_{1}, r_{2}\right)=\frac{c}{r_{2}-r_{1}}\left(\left(r_{2}^{N}-r_{1}^{N}\right) \frac{\pi^{N / 2}}{\Gamma(1+N / 2)}\right)^{1 / p} .
$$

We formulate our main result as follows:

Theorem 1. Let $f: \Omega \times R \rightarrow R$ be an $L^{1}$-Carathéodory function, and denote $F(x, t)=\int_{0}^{t} f(x, \xi) d \xi$ for each $(x, t) \in \Omega \times R$. Assume that there exist three positive constants $\theta, \tau$ and $\gamma$ with $k_{1} \tau>\theta, \gamma<p$ and a function $\mu \in L^{1}(\Omega)_{+}$ such that

$\left(\alpha_{1}\right) F(x, t) \geqslant 0$ for each $(x, t) \in\left(\Omega \backslash S\left(x^{0}, r_{1}\right)\right) \times[0, \tau]$,

$\left(\alpha_{2}\right) \int_{\Omega} \sup _{t \in[-\theta, \theta]} F(x, t) d x<\frac{1}{2}\left(\frac{\theta}{k_{1} \tau}\right)^{p} \int_{S\left(x^{0}, r_{1}\right)} F(x, \tau) d x$,

$\left(\alpha_{3}\right) F(x, t) \leqslant \mu(x)\left(1+|t|^{\gamma}\right)$ for almost every $x \in \Omega$ and for all $t \in R$, 
where $k_{1}$ is given in (2.1). Then, for each

$$
\begin{aligned}
\lambda \in \Lambda_{1}^{\prime}= & ] \frac{\frac{1}{p}\left(\frac{k_{1} \tau}{c}\right)^{p}}{\int_{S\left(x^{0}, r_{1}\right)} F(x, \tau) d x-\int_{\Omega} \sup _{t \in[-\theta, \theta]} F(x, t) d x}, \\
& \frac{\frac{1}{p}\left(\frac{\theta}{c}\right)^{p}}{\int_{\Omega} \sup _{t \in[-\theta, \theta]} F(x, t) d x}[,
\end{aligned}
$$

the problem (1.1) admits at least three weak solutions in $X$ and, moreover, for each $h>1$, there exist an open interval

$$
\Lambda_{2}^{\prime} \subseteq\left[0, \frac{\frac{h}{p}\left(\frac{\theta}{c}\right)^{p}}{\left(\frac{\theta}{k_{1} \tau}\right)^{p} \int_{S\left(x^{0}, r_{1}\right)} F(x, \tau) d x-\int_{\Omega} \sup _{t \in[-\theta, \theta]} F(x, t) d x}\right]
$$

and a positive real number $\sigma$ such that, for each $\lambda \in \Lambda_{2}^{\prime}$, the problem (1.1) admits at least three weak solutions in $X$ whose norms are less than $\sigma$.

Proof. In order to apply Theorem A, we begin by setting

$$
\Phi(u)=\frac{\|u\|^{p}}{p}, \quad J(u)=\int_{\Omega} F(x, u(x)) d x
$$

for each $u \in X$. It is well known that $J$ is a continuously Gâteaux differentiable functional whose Gâteaux derivative at the point $u \in X$ is the functional $J^{\prime}(u) \in X^{*}$, given by

$$
J^{\prime}(u)(v)=\int_{\Omega} f(x, u(x)) v(x) d x
$$

for every $v \in X$. We claim that $J^{\prime}: X \rightarrow X^{*}$ is a compact operator. To this end, it is enough to show that $J^{\prime}$ is strongly continuous on $X$. For this, for fixed $u \in X$ let $u_{n} \rightarrow u$ weakly in $X$ as $n \rightarrow+\infty$, then we have $u_{n}$ converges uniformly to $u$ on $\Omega$ as $n \rightarrow+\infty$ (see [17]). Since $F(x, \cdot)$ is $C^{1}$ in $R$ for every $x \in \Omega$, so it is continuous in $R$ for every $x \in \Omega$, and we get that $F\left(x, u_{n}\right) \rightarrow F(x, u)$ strongly as $n \rightarrow+\infty$ which follows $J^{\prime}\left(u_{n}\right) \rightarrow J^{\prime}(u)$ strongly as $n \rightarrow+\infty$. Thus we proved that $J^{\prime}$ is strongly continuous on $X$, which implies that $J^{\prime}$ is a compact operator by Proposition 26.2 of [19]. Hence the claim is true.

Moreover, the functional $\Phi$ is a continuously Gâteaux differentiable whose Gâteaux derivative at the point $u \in X$ is the functional $\Phi^{\prime}(u) \in X^{*}$, given by

$$
\Phi^{\prime}(u)(v)=\int_{\Omega}|\nabla u(x)|^{p-2} \nabla u(x) \nabla v(x) d x .
$$

$\Phi^{\prime}$ admits a continuous inverse on $X^{*}$. Indeed, owing to (2.2) of [17], for every $u, v \in X$ there exists a positive constant $c_{p}$ such that

$$
\left\langle|\nabla u(x)|^{p-2} \nabla u(x)-|\nabla v(x)|^{p-2} \nabla v(x), \nabla u(x)-\nabla v(x)\right\rangle \geqslant c_{p}|\nabla u(x)-\nabla v(x)|^{p}
$$

where $\langle\cdot, \cdot\rangle$ denotes the usual inner product in $R$. So, we have

$$
\left(\Phi^{\prime}(u)-\Phi^{\prime}(v)\right)(u-v) \geqslant c_{p}\|u-v\|^{p}
$$


for every $u, v \in X$, namely $\Phi^{\prime}$ is an uniformly monotone operator in $X$, and since $\Phi$ is coercive and hemicontinuous in $X$, by applying Theorem 26.A. [19], we have that $\Phi^{\prime}$ admits a continuous inverse on $X^{*}$. Using again that $\Phi^{\prime}$ is monotone, we obtain that $\Phi$ is sequentially weakly lower semi continuous (see [19, Proposition 25.20]).

Thanks to $\left(\alpha_{3}\right)$, for each $\lambda>0$ one has that

$$
\lim _{\|u\| \rightarrow+\infty}(\Phi(u)-\lambda J(u))=+\infty .
$$

Now, set

$$
u^{*}(x)= \begin{cases}0, & x \in \Omega \backslash S\left(x^{0}, r_{2}\right) \\ \frac{\tau}{r_{2}-r_{1}}\left[r_{2}-\sqrt{\sum_{i=1}^{N}\left(x_{i}-x_{i}^{0}\right)^{2}}\right], & x \in S\left(x^{0}, r_{2}\right) \backslash S\left(x^{0}, r_{1}\right) \\ \tau, & x \in S\left(x^{0}, r_{1}\right)\end{cases}
$$

and $r=\frac{1}{p}\left(\frac{\theta}{c}\right)^{p}$. It is easy to see that $u^{*} \in X$ and, in particular, one has

$$
\Phi\left(u^{*}\right)=\frac{1}{p}\left(r_{2}^{N}-r_{1}^{N}\right) \frac{\pi^{N / 2}}{\Gamma(1+N / 2)}\left(\frac{\tau}{r_{2}-r_{1}}\right)^{p} .
$$

So, since $k_{1} \tau>\theta$, we have $\Phi\left(u^{*}\right)>r$. Moreover, since

$$
\sup _{x \in \Omega}|u(x)| \leqslant c\|u\|
$$

for each $u \in X$, one has

$$
\frac{\sup }{u \in \Phi^{-1}(]-\infty, r[)} w(u)=\sup _{\left.\left.u \in \Phi^{-1}(]-\infty, r\right]\right)} J(u) \leqslant \int_{\Omega} \sup _{t \in[-\theta, \theta]} F(x, t) d x,
$$

and since $0 \leqslant u^{*}(x) \leqslant \tau$ for each $x \in \Omega$, the condition $\left(\alpha_{1}\right)$ ensures that

$$
\int_{\Omega \backslash S\left(x^{0}, r_{2}\right)} F\left(x, u^{*}(x)\right) d x+\int_{S\left(x^{0}, r_{2}\right) \backslash S\left(x^{0}, r_{1}\right)} F\left(x, u^{*}(x)\right) d x \geqslant 0 .
$$

Therefore, owing to our assumptions, we have

$$
\begin{aligned}
& \frac{\sup }{u \in \Phi^{-1}(]-\infty, r[)} w(u)=\sup _{\|u\|^{p} \leqslant p r} \int_{\Omega} F(x, u(x)) d x \\
& \leqslant \int_{\Omega} \sup _{|t| \leqslant \theta} F(x, t) d x<\frac{1}{2}\left(\frac{\theta}{k_{1} \tau}\right)^{p} \int_{S\left(x^{0}, r_{1}\right)} F(x, \tau) d x \\
& \leqslant \frac{\frac{1}{p}\left(\frac{\theta}{c}\right)^{p}}{\frac{1}{p}\left(\frac{\theta}{c}\right)^{p}+\frac{1}{p}\left(\frac{k_{1} \tau}{c}\right)^{p}} \int_{S\left(x^{0}, r_{1}\right)} F(x, \tau) d x \leqslant \frac{r}{r+\Phi\left(u^{*}\right)} J\left(u^{*}\right) .
\end{aligned}
$$

Now, we can apply Theorem A. Taking into account that

$$
\begin{aligned}
& \Phi\left(u^{*}\right) /\left(J\left(u^{*}\right)-\sup _{x \in \Phi^{-1}(]-\infty, r[)} w\left(u^{*}\right)\right) \\
& \leqslant \frac{\frac{1}{p}\left(\frac{k_{1} \tau}{c}\right)^{p}}{\int_{S\left(x^{0}, r_{1}\right)} F(x, \tau) d x-\int_{\Omega} \sup _{t \in[-\theta, \theta]} F(x, t) d x}
\end{aligned}
$$




$$
\begin{gathered}
\frac{r}{\sup _{u \in \bar{\Phi}^{-1}(]-\infty, r[)} w(u)} \geqslant \frac{\frac{1}{p}\left(\frac{\theta}{c}\right)^{p}}{\int_{\Omega} \sup _{t \in[-\theta, \theta]} F(x, t) d x} ; \\
\frac{h r}{r \frac{J\left(u^{*}\right)}{\Phi\left(u^{*}\right)}-\sup _{u \in \bar{\Phi}^{-1}(-\infty, r[)} w(u)} \\
\leqslant \frac{\frac{h}{p}\left(\frac{\theta}{c}\right)^{p}}{\left(\frac{\theta}{k_{1} \tau}\right)^{p} \int_{S\left(x^{0}, r_{1}\right)} F(x, \tau) d x-\int_{\Omega} \sup _{t \in[-\theta, \theta]} F(x, t) d x}=\rho ;
\end{gathered}
$$

and with $x_{0}=0, x_{1}=u^{*}$, and see $\Lambda_{1}^{\prime} \subseteq \Lambda_{1}, \Lambda_{2} \subseteq \Lambda_{2}^{\prime}$, and also taking into account that the weak solutions of the problem (1.1) are exactly the solutions of the equation

$$
\Phi^{\prime}(u)-\lambda J^{\prime}(u)=0,
$$

from Theorem $\mathrm{A}$ it follows that, for each $\lambda \in \Lambda_{1}^{\prime}$, the problem (1.1) admits at least three weak solutions, and there exist an open interval $\Lambda_{2}^{\prime} \subseteq[0, \rho]$ and a real positive number $\sigma$ such that, for each $\lambda \in \Lambda_{2}^{\prime}$, the problem (1.1) admits at least three weak solutions that whose norms in $\mathrm{X}$ are less than $\sigma$. Hence, we have the conclusion.

Remark 1. In Theorem 1,

$$
\frac{\frac{1}{p}\left(\frac{k_{1} \tau}{c}\right)^{p}}{\int_{S\left(x^{0}, r_{1}\right)} F(x, \tau) d x-\int_{\Omega} \sup _{t \in[-\theta, \theta]} F(x, t) d x}<\frac{\frac{1}{p}\left(\frac{\theta}{c}\right)^{p}}{\int_{\Omega} \sup _{t \in[-\theta, \theta]} F(x, t) d x} .
$$

Because, from $\left(\alpha_{2}\right)$ we have

$$
2\left(k_{1} \tau\right)^{p} \int_{\Omega} \sup _{t \in[-\theta, \theta]} F(x, t) d x<\theta^{p} \int_{S\left(x^{0}, r_{1}\right)} F(x, \tau) d x,
$$

and since $k_{1} \tau>\theta$, we get

$$
\left(\theta^{p}+\left(k_{1} \tau\right)^{p}\right) \int_{\Omega} \sup _{t \in[-\theta, \theta]} F(x, t) d x<\theta^{p} \int_{S\left(x^{0}, r_{1}\right)} F(x, \tau) d x,
$$

and so

$$
\left(k_{1} \tau\right)^{p} \int_{\Omega} \sup _{t \in[-\theta, \theta]} F(x, t) d x<\theta^{p}\left(\int_{S\left(x^{0}, r_{1}\right)} F(x, \tau) d x-\int_{\Omega} \sup _{t \in[-\theta, \theta]} F(x, t) d x\right) .
$$

Hence, multiplying by $\frac{1}{p c^{p}}$ we obtain

$$
\begin{aligned}
& \frac{1}{p}\left(\frac{k_{1} \tau}{c}\right)^{p} \int_{\Omega} \sup _{t \in[-\theta, \theta]} F(x, t) d x \\
& \quad<\frac{1}{p}\left(\frac{\theta}{c}\right)^{p}\left(\int_{S\left(x^{0}, r_{1}\right)} F(x, \tau) d x-\int_{\Omega} \sup _{t \in[-\theta, \theta]} F(x, t) d x\right),
\end{aligned}
$$


which follows

$$
\frac{\frac{1}{p}\left(\frac{k_{1} \tau}{c}\right)^{p}}{\int_{S\left(x^{0}, r_{1}\right)} F(x, \tau) d x-\int_{\Omega} \sup _{t \in[-\theta, \theta]} F(x, t) d x}<\frac{\frac{1}{p}\left(\frac{\theta}{c}\right)^{p}}{\int_{\Omega} \sup _{t \in[-\theta, \theta]} F(x, t) d x} .
$$

Remark 2. In applying Theorem 1, it is enough to know as explicit upper bound of the constant $c$. To be precise, we can use formula (2.1) as constant $c$ the righthand term of the formula in page 393 , so that the constant $k_{1}$ in Theorem 1 is numerically well determined.

We now present a particular case of Theorem 1 , in which the function $f$ has separated variables.

Corollary 1. Let $f_{1} \in L^{1}(\Omega)$ and $f_{2} \in C(R)$ be two functions. Put $\widetilde{F}(t)=$ $\int_{0}^{t} f_{2}(\xi) d \xi$ for all $t \in R$, and assume that there exist four positive constants $\theta$, $\tau, \eta$ and $\gamma$ with $k_{1} \tau>\theta, \gamma<p$ such that

$\left(\alpha_{1}^{\prime}\right) f_{1}(x) \geqslant 0$ for each $x \in \Omega \backslash S\left(x^{0}, r_{1}\right)$ and $f_{2}(t) \geqslant 0$ for each $t \in[0, \tau]$,

$\left(\alpha_{2}^{\prime}\right) \max _{t \in[-\theta, \theta]} \widetilde{F}(t)\left(\int_{\Omega} f_{1}(x) d x\right)<\frac{\widetilde{F}(\tau)}{2}\left(\frac{\theta}{k_{1} \tau}\right)^{p} \int_{S\left(x^{0}, r_{1}\right)} f_{1}(x) d x$,

$\left(\alpha_{3}^{\prime}\right)|\widetilde{F}(t)| \leqslant \eta\left(1+|t|^{\gamma}\right)$ for all $t \in R$,

where $k_{1}$ is given in (2.1). Then, for each

$$
\begin{aligned}
\lambda \in \Lambda_{1}^{\prime}= & \frac{\frac{1}{p}\left(\frac{k_{1} \tau}{c}\right)^{p}}{\widetilde{F}(\tau) \int_{S\left(x^{0}, r_{1}\right)} f_{1}(x) d x-\max _{|t| \leqslant \theta} \widetilde{F}(t)\left(\int_{\Omega} f_{1}(x) d x\right)}, \\
& \frac{\frac{1}{p}\left(\frac{\theta}{c}\right)^{p}}{\max _{|t| \leqslant \theta} \widetilde{F}(t)\left(\int_{\Omega} f_{1}(x) d x\right)}[
\end{aligned}
$$

the problem

$$
\begin{cases}\Delta_{p} u+\lambda f_{1}(x) f_{2}(u)=0 & \text { in } \Omega \\ u=0 & \text { on } \partial \Omega,\end{cases}
$$

admits at least three weak solutions in $X$ and, moreover, for each $h>1$, there exists an open interval

$$
\Lambda_{2}^{\prime} \subseteq\left[0, \frac{\frac{h}{p}\left(\frac{\theta}{c}\right)^{p}}{\left(\frac{\theta}{k_{1} \tau}\right)^{p} \widetilde{F}(\tau) \int_{S\left(x^{0}, r_{1}\right)} f_{1}(x) d x-\max _{|t| \leqslant \theta} \widetilde{F}(t)\left(\int_{\Omega} f_{1}(x) d x\right)}\right]
$$

and a positive real number $\sigma$ such that, for each $\lambda \in \Lambda_{2}^{\prime}$, the problem (2.2) admits at least three weak solutions in $X$ whose norms are less than $\sigma$.

Proof. Set $f(x, u)=f_{1}(x) f_{2}(u)$ for each $(x, u) \in \Omega \times R$. Since

$$
F(x, t)=f_{1}(x) \widetilde{F}(t),
$$

from $\left(\alpha_{1}^{\prime}\right)$ and $\left(\alpha_{2}^{\prime}\right)$ we obtain $\left(\alpha_{1}\right)$ and $\left(\alpha_{2}\right)$, respectively. From $(2.3)$ and $\left(\alpha_{3}^{\prime}\right)$ we have

$$
F(x, t) \leqslant\left|f_{1}(x) \widetilde{F}(t)\right| \leqslant \eta\left|f_{1}(x)\right|\left(1+|t|^{\gamma}\right)
$$

for each $(x, t) \in \Omega \times R$, so condition $\left(\alpha_{3}\right)$ follows with $\mu(x)=\eta\left|f_{1}(x)\right|$. Then, Theorem 1 yields the conclusion. 
Example 1. Consider the problem

$$
\begin{cases}\operatorname{div}(|\nabla u| \nabla u)+\lambda\left(e^{-u} u^{10}(11-u)\right)=0 & \text { in } \Omega \\ u=0 & \text { on } \partial \Omega\end{cases}
$$

where $\Omega=\left\{(x, y) \in R^{2} ; x^{2}+y^{2}<9\right\}$. Taking into account $c=\sqrt[6]{36 / \pi^{2}}$, choosing $x^{0}=(0,0), r_{1}=1, r_{2}=2, f_{1}(x)=1$ for all $x \in \Omega$ and

$$
f_{2}(u)=e^{-u} u^{10}(11-u)
$$

for each $u \in R$, so that $k_{1}=\sqrt[6]{324}$, all the assumptions of Corollary 1, with $p=3$, are satisfied by choosing, for instance $\theta=1, \tau=3, \gamma=2$ and $\eta$ sufficiently large. So for each $\lambda \in] \frac{3 e}{3^{9} e^{-2}-1}, \frac{e}{162}[$, the problem (2.4) admits at least three non-trivial weak solutions in $W_{0}^{1,3}(\Omega)$ and, moreover, for each $h>1$, there exist an open interval $\Lambda \subseteq] 0, \frac{h e}{9\left(729 e^{-2}-18\right)}[$ and a positive real number $\sigma$ such that, for each $\lambda \in \Lambda$, the problem (2.4) admits at least three weak solutions in $W_{0}^{1,3}(\Omega)$ whose norms are less than $\sigma$.

Finally, we want to point out a simple consequence of Theorem 1 in the ordinary case with $p=2$, and then we present an example of application.

For simplicity, we fix $\Omega=(a, b)$ for $a, b \in R$ and $x^{0} \in \Omega$. Taking into account that, in this situation, $c=\frac{(b-a)^{\frac{1}{2}}}{2}, k_{1}=\left(\frac{b-a}{2\left(r_{2}-r_{1}\right)}\right)^{\frac{1}{2}}$ and $k_{2}=$ $\frac{1}{2}\left(\frac{b-a}{r_{1}\left(r_{2}-r_{1}\right)}\right)^{\frac{1}{2}}$, we have the following result:

Corollary 2. Let $f:[a, b] \times R \rightarrow R$ be a continuous function and put $F(x, t)=$ $\int_{0}^{t} f(x, \xi) d \xi$ for each $(x, t) \in[a, b] \times R$. Assume that there exist three positive constants $\theta, \tau$ and $\gamma$ with $\left(\frac{b-a}{2\left(r_{2}-r_{1}\right)}\right)^{\frac{1}{2}} \tau>\theta, \gamma<2$ and a function $\mu \in L^{1}([a, b])_{+}$ such that

$$
\begin{aligned}
& \left(\alpha_{1}^{\prime \prime}\right) F(x, t) \geqslant 0 \text { for each }(x, t) \in\left((a, b) \backslash\left(x^{0}-r_{1}, x^{0}+r_{1}\right)\right) \times[0, \tau], \\
& \left(\alpha_{2}^{\prime \prime}\right) \int_{a}^{b} \sup _{t \in[-\theta, \theta]} F(x, t) d x<\frac{r_{2}-r_{1}}{b-a}\left(\frac{\theta}{\tau}\right)^{2} \int_{x^{0}-r_{1}}^{x^{0}+r_{1}} F(x, \tau) d x, \\
& \left(\alpha_{3}^{\prime \prime}\right) F(x, t) \leqslant \mu(x)\left(1+|t|^{\gamma}\right) \text { for almost every } x \in(a, b) \text { and for all } t \in R .
\end{aligned}
$$

Then, for each

$$
\begin{aligned}
\lambda \in \Lambda_{1}^{\prime}= & ] \frac{\tau^{2} /\left(r_{2}-r_{1}\right)}{\int_{x^{0}-r_{1}}^{x^{0}+r_{1}} F(x, \tau) d x-\int_{a}^{b} \sup _{t \in[-\theta, \theta]} F(x, t) d x}, \\
& \frac{2 \theta^{2}}{(b-a) \int_{a}^{b} \sup _{t \in[-\theta, \theta]} F(x, t) d x}[
\end{aligned}
$$

the problem

$$
\left\{\begin{array}{l}
u^{\prime \prime}+\lambda f(x, u)=0 \quad \text { in }(a, b) \\
u(a)=u(b)=0
\end{array}\right.
$$

admits at least three weak solutions in $X$ and, moreover, for each $h>1$, there exists an open interval

$$
\Lambda_{2}^{\prime} \subseteq\left[0, \frac{2 h \theta^{2}}{2\left(r_{2}-r_{1}\right)\left(\frac{\theta}{\tau}\right)^{2} \int_{x^{0}-r_{1}}^{x^{0}+r_{1}} F(x, \tau) d x-(b-a) \int_{a}^{b} \sup _{t \in[-\theta, \theta]} F(x, t) d x}\right]
$$


and a positive real number $\sigma$ such that, for each $\lambda \in \Lambda_{2}^{\prime}$, the problem (2.5) admits at least three classical solutions in $X$ whose norms are less than $\sigma$.

Example 2. Put

$$
f(x, u)=e^{-(x+u)} u^{6}(7-u)
$$

for each $(x, u) \in(-3,3) \times R$, and choose $x^{0}=0, r_{1}=1, r_{2}=2$. It is easy to verify that with $\theta=1, \tau=3, \gamma=1$ and $\mu(x)$ for each $x \in(-3,3)$ sufficiently large, all the assumptions of Corollary 2 , are satisfied. So for each $\lambda \in] \frac{9}{2187\left(e^{-2}-e^{-4}\right)+e^{-4}-e^{2}}, \frac{1}{3\left(e^{2}-e^{-4}\right)}[$, the problem

$$
\left\{\begin{array}{l}
u^{\prime \prime}+\lambda\left(e^{-(x+u)} u^{6}(7-u)\right)=0 \quad \text { in }(-3,3), \\
u(-3)=u(3)=0
\end{array}\right.
$$

admits at least three non-trivial classical solutions in $W_{0}^{1,2}([-3,3])$ and, moreover, for each $h>1$, there exist an open interval $\Lambda \subseteq] 0, \frac{h}{32\left(e^{-2}-e^{-4}\right)-3\left(e^{2}-e^{-4}\right)}[$ and a positive real number $\sigma$ such that, for each $\lambda \in \Lambda$, the problem (2.6) admits at least three classical solutions in $W_{0}^{1,2}([-3,3])$ whose norms are less than $\sigma$.

Remark 3. The weak solutions of the problem (1.1) where $f$ is a continuous function, in the ordinary case with $\Omega=(a, b), a, b \in R$ and $p=2$, by using standard methods, belong to $C^{2}([a, b])$ and are classical solutions for the problem (1.1). Namely, in this case, the classical and the weak solutions of the problem (1.1) coincide.

\section{Acknowledgement}

The authors would like to thank professor D. Motreanu for valuable suggestions. The authors also express their sincere gratitude to the referees for their useful comments and suggestions concerning improvement of this paper.

\section{References}

[1] G.A. Afrouzi and S. Heidarkhani. Three solutions for a Dirichlet boundary value problem involving the $p$-Laplacian. Nonlinear Anal., 66:2281-2288, 2007. Doi:10.1016/j.na.2006.03.019.

[2] D. Averna and G. Bonanno. A three critical points theorem and its applications to the ordinary Dirichlet problem. Topol. Methods Nonlinear Anal., 22:93-104, 2003.

[3] R.I. Avery and J. Henderson. Three symmetric positive solutions for a second-order boundary value problem. Appl. Math. Lett., 13:1-7, 2000. Doi:10.1016/S0893-9659(99)00177-9.

[4] G. Bonanno. Existence of three solutions for a two point boundary value problem. Appl. Math. Lett., 13:53-57, 2000. Doi:10.1016/S0893-9659(00)00033-1.

[5] G. Bonanno. Some remarks on a three critical points theorem. Nonlinear Anal., 54:651-665, 2003. Doi:10.1016/S0362-546X(03)00092-0. 
[6] G. Bonanno. A critical points theorem and nonlinear differential problems. $J$. Global Optim., 28:249-258, 2004. Doi:10.1023/B:JOGO.0000026447.51988.f6.

[7] G. Bonanno and R. Livrea. Multiplicity theorems for the Dirichlet problem involving the $p$-Laplacian. Nonlinear Anal., 54:1-7, 2003. Doi:10.1016/S0362-546X(03)00027-0.

[8] F. Cammaroto, A. Chinni' and B. Di Bella. Multiple solutions for a Dirichlet problem involving the p-Laplacian. Dynam. Systems Appl., 16:673-680, 2007.

[9] P. Drabek and J. Hernandez. Existence and uniqueness of positive solutions for some quasilinear elliptic problems. Nonlinear Anal., 44:189-204, 2001. Doi:10.1016/S0362-546X(99)00258-8.

[10] Z.M. Guo. On the number of positive solutions for quasilinear elliptic equations when a parameter is large. Nonlinear Anal., 27:229-247, 1996. Doi:10.1016/0362-546X(94)00352-I.

[11] Z.M. Guo and J.R.L. Webb. Uniqueness of positive solutions for quasilinear elliptic equations when a parameter is large. Proc. Roy. Soc. Edinburgh, 124:189198, 1994.

[12] D.D. Hai and R. Shivaji. Existence and uniqueness for a class of quasilinear elliptic boundary value problems. J. Differential Equations, 193:500-510, 2003. Doi:10.1016/S0022-0396(03)00028-7.

[13] S.A. Marano and D. Motreanu. On a three critical points theorem for nondifferentiable functions and applications nonlinear boundary value problems. Nonlinear Anal., 48:37-52, 2002. Doi:10.1016/S0362-546X(00)00171-1.

[14] M. Ramaswamy and R. Shivaji. Multiple positive solutions for classes of $p$ Laplacian equations. Differ. Integral Equ., 17:1255-1261, 2004.

[15] B. Ricceri. On a three critical points theorem. Arch. Math. (Basel), 75:220-226, 2000.

[16] B. Ricceri. A three critical points theorem revisited. Nonlinear Anal., 70:30843089, 2009. Doi:10.1016/j.na.2008.04.010.

[17] J. Simon. Regularitè de la Solution d'une Equation Non lineaire dans $R^{N}$. LMN 665. P. Benilan ed., Berlin, Heidelberg, New York, 1978.

[18] G. Talenti. Some inequalities of Sobolev type on two-dimensional spheres. in: $W$. Walter (Ed.), General Inequalities, Vol. 5, Internat. Ser Numer. Math., 8:401408, 1987.

[19] E. Zeidler. Nonlinear Functional Analysis and Its Applications. Vol. II/B. Berlin, Heidelberg, New York, 1985. 